DARMKREBSMONAT MÄRZ

\section{Motivieren Sie zur Vorsorge!}

Zahlreiche Aktionen informieren jedes Jahr im März über das Thema Darmkrebs. Damit sollen mehr Menschen motiviert werden, an der Darmkrebsfrüherkennung teilzunehmen. Eine Studie des DKFZ hat erst kürzlich ergeben: Bei denjenigen, die in den letzten zehn Jahren an einer Darmspiegelung teilgenommen hatten, traten $48 \%$ weniger fortgeschrittene Darmkrebsvorstufen auf als bei denen, die zum ersten Mal eine Darmspiegelung durchführen ließen.

\section{Info-Hotline}

Infos zum Darmkrebs für Patienten, Angehörige und Ärzte: Telefon täglich 8-20 Uhr kostenfrei: 0800-4203040, E-Mail: krebsinformation@dkzf.de, www.krebsinformationsdienst.de

\section{RKI MELDET}

\section{Anstieg bei Hantavirus-Infektionen}

In den ersten fünf Wochen dieses Jahres wurden bereits 101 Hantavirus-Infektionen an das Robert Koch-Institut gemeldet, vorwiegend aus Baden-Württemberg (62 Fälle). Damit könnte sich eine ähnlich hohe Infektionsrate wie 2007 ankündigen (1721 Fälle).

In Deutschland wird der vorherrschende Virustyp Puumala vor allem durch Speichel, Kot und Urin der Rötelmaus übertragen. Die Erkrankung beginnt nach einer Inkubationszeit von zwei bis vier Wochen meist abrupt mit hohem Fieber, das über drei bis vier Tage anhält. Hinzu kommen grippeähnliche Symptome wie Kopf-, Abdominalschmerzen und Myalgien. Bei einem Teil der Patienten entwickelt sich eine dialysepflichtige Niereninsuffizienz, die aber reversibel ist. Gefährdet sind vor allem Personen in Endemiegebieten (z.B. Schwäbische Alb mit an-

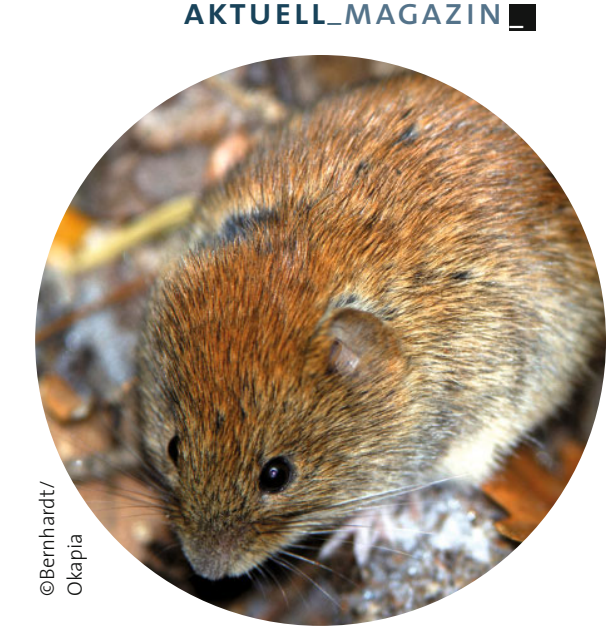

Hauptüberträger der Hantaviren: die Rötelmaus.

grenzenden Gebieten, Spessart), die zu Mäusen und deren Ausscheidungen Kontakt haben. Da es weder Impfung noch spezifische Therapie gibt, sind Präventionsmaßnahmen wichtig. Bevor potenziell kontaminierte Bereiche (z B. Keller, Dachböden) betreten werden, sollte gut gelüftet werden. Beim Reinigen der Bereiche darf kein Staub aufgewirbelt oder sollten Atemschutzmasken getragen werden. Merkblätter unter: www.rki.de (unter Infektionskrankheiten A-Z). KE .

\title{
REISEMEDIZIN
}

\section{Malaria oder Montezumas Rache: Das Geschlecht entscheidet mit}

Montezuma scheint auf Frauen schlecht zu sprechen zu sein: Wie Forscher vom Zentrum für Reisemedizin der Universität Zürich herausgefunden haben, erkranken Angehörige des weiblichen Geschlechts häufiger an einer Reisediarrhö als Männer; das gilt sowohl für die akute als auch für die chronische Form.

Um den Zusammenhang zwischen Geschlecht und Reisekrankheiten aufzudecken, waren Daten von knapp 60000 Patientinnen und Patienten aus den Jahren 1997-2007 ausgewertet worden. Der „kleine Unterschied“ spielt demnach nicht nur bei gastrointestinalen Problemen eine Rolle. Frauen auf Reisen leiden häufiger an Zahnschmerzen, Infektionen der oberen Atemwege und Medikamentenunverträglichkeiten. Männer fangen sich dafür öfter sexuell übertragbare Erkrankungen oder Hepatitis ein; sie dringen in extremere Gefilde vor, wo sie akute Höhenkrankheit oder Erfrierungen erwerben, und landen häufiger im Krankenhaus. Während sich diese Unterschiede mit der größeren Risikobereitschaft des „starken“
Geschlechts erklären lassen, liegt die Sache bei der Malaria (von der ebenfalls Männer häufiger betroffen sind) anders: Mücken fliegen offenbar verstärkt auf die Herren der Schöpfung; dies liegt daran, dass Männer mehr von den für die Blutsauger attraktiven Duftstoffen produzieren.

Die unterschiedliche Anfälligkeit der Geschlechter müsste in der

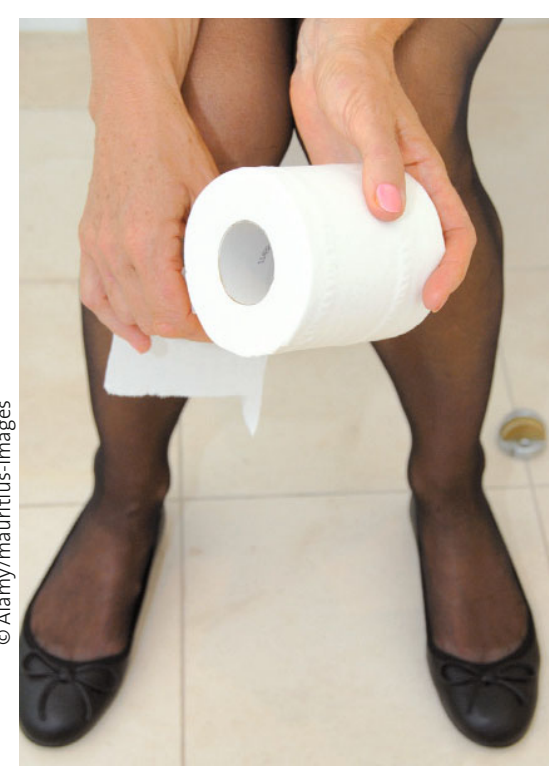
reisemedizinischen Beratung Berücksichtigung finden, fordern die Studienautoren. Frauen sollten verstärkt mit Mitteln zur Selbstbehandlung von Harnwegsinfekten und Diarrhö versorgt werden, Männer müssten mehr Informationen zum Schutz vor Moskitos und zur Prävention sexuell übertragbarer Erkrankungen erhalten. Letzteres wird allerdings durch einen weiteren geschlechtsspezifischen Unterschied erschwert: Männer holen vor Reiseantritt deutlich seltener den Rat des Arztes ein als Frauen. EO Clin Inf Dis 2010;50:826-832, DOI: 10.1086/650575

4 Für Reisediarrhö sind Frauen anfälliger als Männer. 